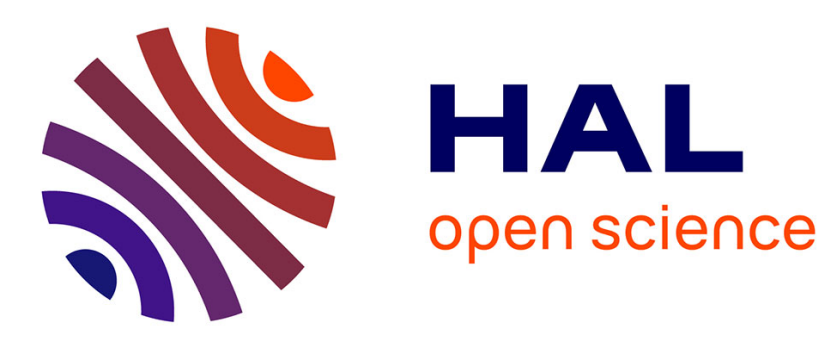

\title{
Advanced backside sample preparation for multi-technique surface analysis
}

\author{
M. Py, M. Veillerot, J.M. Fabbri, F. Pierre, D. Jalabert, C. Roukoss, B. \\ Pelissier, R. Boujamaa, C. Trouiller, J.P. Barnes
}

\section{- To cite this version:}

M. Py, M. Veillerot, J.M. Fabbri, F. Pierre, D. Jalabert, et al.. Advanced backside sample preparation for multi-technique surface analysis. European Physical Journal: Applied Physics, 2011, 55 (3), 10.1051/epjap/2011110191 . hal-00724133

\section{HAL Id: hal-00724133 \\ https://hal.science/hal-00724133}

Submitted on 18 Aug 2012

HAL is a multi-disciplinary open access archive for the deposit and dissemination of scientific research documents, whether they are published or not. The documents may come from teaching and research institutions in France or abroad, or from public or private research centers.
L'archive ouverte pluridisciplinaire HAL, est destinée au dépôt et à la diffusion de documents scientifiques de niveau recherche, publiés ou non, émanant des établissements d'enseignement et de recherche français ou étrangers, des laboratoires publics ou privés. 


\title{
TITLE
}

\section{Advanced backside sample preparation for multi-technique surface analysis}

\author{
AUTHORS \\ M. Py ${ }^{1 *}$, M. Veillerot ${ }^{1}$, J.M. Fabbri ${ }^{1}$, F. Pierre ${ }^{1}$, D. Jalabert ${ }^{2}$, C. Roukoss ${ }^{3}$, B. Pelissier ${ }^{3}$, R. \\ Boujamaa $^{1,4}$, C. Trouiller ${ }^{4}$, J.P. Barnes ${ }^{1}$ \\ ${ }^{1}$ CEA-Leti, MINATEC Campus, 17 rue des Martyrs, 38054 GRENOBLE Cedex 9, France \\ ${ }^{2}$ CEA-INAC/UJF-Grenoble1 UMR-E, SP2M, LEMMA, Minatec Grenoble, F-38054 \\ ${ }^{3}$ LTM-CNRS/CEA-Leti, 17 rue des Martyrs, 38054 GRENOBLE Cedex 9, France \\ ${ }^{4}$ STMicroelectronics, 850 rue de Jean Monnet, 38926 Crolles, France \\ *e-mail : matthieu.py@cea.fr
}

\begin{abstract}
Backside sample preparation is a well known method to help circumvent undesired effects and artifacts in the analysis of a sample or device structure. However it remains challenging in the case of thin layers analysis since only a fraction of the original sample must remain while removing most or all of the substrate and maintaining a smooth and flat surface suitable for analysis.

Here we present a method adapted to the preparation of ultra thin layers grown on pure $\mathrm{Si}$ substrates. It consists in a mechanical polishing up to a few remaining microns, followed by a dedicated wet etch. This method can be operated in a routine fashion and yields an extremely flat and smooth surface, without any remaining Si from substrate. It therefore allows precise analysis of the layers of interests with various characterization techniques.
\end{abstract}

\section{INTRODUCTION}

Over the past decade it has become increasingly common to resort to backside analysis to overcome weaknesses such as ion mixing and preferential sputtering in Secondary Ion Mass Spectrometry (SIMS) depth profiling, or to bring the layer of interest as close as possible to the surface for X-ray Photoelectron Spectroscopy (XPS) analysis [1-6]. With the progress upon the $32 \mathrm{~nm}$ and below nodes for microelectronics, the emergence of new materials in the standard layer stack combined with the aggressive dimensional scaling increase the difficulty of drawing accurate conclusions from a conventional front-side analysis [7-8]. Backside preparation is one of the solutions which could bring answers to this problem. However in the case of such thin layers, the sample preparation remains challenging. Indeed, starting from the whole sample on thick substrate, only a few tenths of nanometers corresponding to the layers of interest must remain while maintaining a smooth and flat 
surface suitable for analysis. Recent developments have used Silicon on Insulator (SOI) substrates where preparation is performed by selective plasma etch, with the buried thick oxide acting as an etch stop layer [9]. This method, although providing sufficient etch selectivity against metals or oxides, was seemingly designed for unattended fashion preparation. It therefore implies consequent over-etching which prohibits its use for accurate preparation of samples grown on pure Si substrate and without a consequently thick (a few tenths of nanometers or more) etch stop layer. It is thus impractical for the preparation of samples grown on Si substrates and featuring nanometer or sub-nanometer ultrathin pedestal oxides. On the other hand, the work from Hantschel et al. investigates a procedure allowing extremely precise preparation for samples featuring sub-nanometer etch stop layers [6]. However the latter presents several drawbacks: (i) it requires a complex mechanical polishing step, involving counting interference fringes at low remaining Si thicknesses, (ii) the useful area yield per sample is quite small and (iii) the obtained surface quality is not ideal, with a $5 \mathrm{~nm}$ Root Mean Square (RMS) roughness for a $30 \times 30 \mu \mathrm{m}$ Atomic Force Microscopy (AFM) scan.

Here we propose a simple method for backside sample preparation adapted to samples grown on Si substrates and featuring nanometer or sub-nanometer ultrathin pedestal oxides. It consists in a mechanical polishing up to a few remaining microns, followed by a dedicated TetraMethylAmmonium Hydroxide (TMAH) etch. This method has two combined advantages. First it is simple to perform, since high precision is not required for the polishing step (provided that the polisher used for preparation enables semi-automatic operation). Second, it is able to reveal layers of interest even in the case of ultra-thin etch stop layers thanks to the optimized conditions for TMAH etch. We therefore used it to prepare samples consisting of high-k/metal gate stacks for $32 \mathrm{~nm}$ node Complementary Metal Oxide Semiconductor (CMOS) devices. On these samples the etch stop layer is materialized by a $1.5 \mathrm{~nm}$ thick SiON layer. In a first step, the preparation method will be described in detail, along with the resulting sample surface state. The ability of this method to provide Si-less surfaces for direct analysis of the layers of interest will be assessed, along with the amount of remaining SiON thickness. The improvements brought by the sample preparation for multi-technique analysis against conventional frontside analysis will be discussed.

\section{EXPERIMENTAL}

For this study, several full sheet samples representative of the structures of actual high$\mathrm{k} /$ metal gate nMOS devices were prepared on (100) Si wafers. The samples are therefore composed of a stack of heterogeneous layers featuring, starting from the Si substrate, (i) a thin SiON (1.5 nm) layer, (ii) an HfSiON (1.7 nm) layer, (iii) a $0.4 \mathrm{~nm}$ lanthanum oxide layer, (iv) a $6.5 \mathrm{~nm}$ thick TiN gate layer and (v) a $60 \mathrm{~nm}$ thick Poly-Si capping. To deposit $\mathrm{SiON}, \mathrm{SiO}_{2}$ was first formed by oxidation of the silicon substrate with in-situ steam generation at $1025^{\circ} \mathrm{C}$, then nitridized. Hafnium silicate was deposited by Metal Organic Chemical Vapor Deposition (MOCVD) with an industry state of the art fab tool. Tetrakis(diethylamino)hafnium (TDEAH) and tris(dimethylamino)silane (TDMAS) were used as precursors for hafnium and silicon respectively. Nitridation of both pedestal $\mathrm{SiO}_{2}$ and hafnium silicate was carried out by an Inductively Coupled Plasma (ICP) nitridation, followed by a $1000^{\circ} \mathrm{C}$ rapid thermal anneal. $\mathrm{LaO}_{\mathrm{x}}$ capping layers and metal gate were formed by Physical Vapor Deposition (PVD). The following Poly-Si was deposited by Chemical Vapor Deposition (CVD). In order to perform analyses, Poly-Si top layers were chemically etched off with TMAH. Half of the samples were then kept as deposited, while the other half were prepared for backside analysis. 
G2 resin and hardener epoxy glue was used to stick together the sample to analyze and its support wafer. A 20 min anneal at $120^{\circ} \mathrm{C}$ in a dedicated incubator was applied for epoxy curing. Physical polishing was performed with the automatic polisher Centar Frontier from Gatan, using 3M diamond abrasive lapping film disks of 30, 6, 3 and $1 \mu \mathrm{m}$ grade. Prior to wet etch, a protective Pt film was deposited on the sides and on the bottom side of the polished sample by sputter coating using a SC500 metallizer from Biorad. Possibly any other metal resistant to TMAH could be used to perform this step at the condition that its deposition provides satisfactory sample coverage. Finally wet etching was performed using small volumes of a $25 \%$ concentrated TMAH solution heated at $90^{\circ} \mathrm{C}$ on a hot plate under an extraction hood. This ensures fast Si etching rate, smooth surface state and high selectivity versus oxides and metal (5000:1 assumed) [10].

Physical characterization of both unprepared and backside prepared samples was performed using several techniques to investigate the results yielded by the preparation.

Sample surface morphology was observed right after the wet etch step and prior to analysis with other techniques with a Multimode AFM NANOSCOPE III from Digital Instruments in tapping mode.

Time of Flight SIMS (ToF-SIMS) depth profiling was performed with a TOF SIMS V tool from ION-TOF GmbH with a caesium ion source for sputtering and a bismuth liquidmetal ion source for analysis, all beams being incident at approximately $45^{\circ}$. Profiles were acquired by analysis of negative secondary ions. Caesium sputtering was carried out with a $250 \mathrm{eV} \mathrm{Cs}^{+}$beam with a $300 \mu \mathrm{m} \times 300 \mu \mathrm{m}$ raster to allow slow abrasion of the surface ( $1 \mathrm{~nm} \cdot \mathrm{min}^{-1}$ in silicon) and maximize depth resolution close to the surface of the sample. For analysis, a $15 \mathrm{keV} \mathrm{Bi}^{+}$beam was used with a $120 \mu \mathrm{m} \times 120 \mu \mathrm{m}$ raster in the centre part of the sputter crater in order to obtain a reasonable sputter/analysis ratio. Vacuum in the analysis chamber was kept between $7.10^{-8} \mathrm{~Pa}$ and $1.10^{-7} \mathrm{~Pa}$ while profiling to reduce the influence of residual gases (typically oxygen and hydrogen) on the analysis. Finally, sputter rate in pure $\mathrm{Si}$ was measured by profiling a reference sample constituted by multiple boron deltas in a silicon matrix characterized by High Resolution Transmission Electron Microscope [11]. Sputter rate in silicon dioxide was estimated using a thick $\mathrm{SiO}_{2}$ layer measured by ellipsometry.

Medium Energy Ion Scattering (MEIS) experiments were carried out on a $400 \mathrm{kV}$ accelerator from HVEE coupled to an ultrahigh vacuum analysis chamber through a differentially pumped beamline. A toroïdal electrostatic analyser (TEA) combined with a bidimensional position sensitive detector enabled the simultaneous energy and angle detection of the backscattered ions. A $100 \mathrm{keV} \mathrm{He}{ }^{+}$ion beam was used at an incidence angle of $40^{\circ}$, while the backscattered particles were analysed in a $20^{\circ}$ window centred at $110^{\circ}$ with respect to the incident direction. The choice of these experimental parameters was motivated by the need of high depth resolution to discriminate the different Si-based layers. The energy-depth conversion of the RBS energy spectra was performed by computer simulation using tabulated stopping powers for $\mathrm{He}^{+}$ions [12].

Samples were also analyzed with a customized Theta 300 spectrometer from Thermo Fisher Scientific with parallel angle resolved capability, allowing simultaneous acquisition of a wide angle range without sample tilt. The photoelectrons corresponding to the $\mathrm{C}_{1 \mathrm{~s}}, \mathrm{Si}_{2 \mathrm{p}} / \mathrm{La}_{4 \mathrm{~d}}$, $\mathrm{O}_{1 \mathrm{~s}}, \mathrm{~N}_{1 \mathrm{~s}}, \mathrm{Hf}_{4 \mathrm{f}}, \mathrm{Ti}_{2 \mathrm{p}}$ and $\mathrm{La}_{3 \mathrm{~d}}$ core levels were simultaneously acquired from 8 angles (from $23.75^{\circ}$ to $\left.76.25^{\circ}\right)$ using a high resolution monochromatic Al Ka X-ray source $(1486.6 \mathrm{eV}$ photons) and at a pass energy of $100 \mathrm{eV}$. By using a numerical procedure, spectral fitting was performed to extract the peaks contributions in the acquired energy regions. Individual line 
shapes were simulated with a combination of Lorentzian and Gaussians functions. The background subtraction was realized using a Shirley function.

\section{BACKSIDE PREPARATION}

The backside preparation proposed in this work features four main process steps, illustrated in Fig. 1.

The first one consists in the preparation of the sample and of its support substrate see (Fig.1, 1.). Both of them are cleaved approximately $( \pm 0.5 \mathrm{~mm})$ into identical sized pieces using a diamond tip. The pieces dimensions are dictated by the polisher's sample holder compliance, from $3 \mathrm{~mm}$ minimum to $7 \mathrm{~mm}$ maximum in both width and length. The support substrate might be any piece of standard wafer. Yet, for optimized visualization of the preparation advancement in the final etch step it is better if its surface features coloured patterns. However it should not present important topography, which might hinder the final surface quality. In this study, we therefore used a substrate with various metallic lines and patterns evened by Chemical Mechanical Polishing (CMP). The maximum peak-to valley topography of this substrate was found to be of $\pm 50 \mathrm{~nm}$, as measured by contact profilometry. Both pieces are then cleaned in Acetone and IsoPropyl Alcohol (IPA) to remove any surface contamination, and glued together, taking care to avoid gas inclusions in the glue. After what, the four sides of the stack are polished to ensure the absence of cleaving residues, glue traces, particles and edge mismatches on all of them. This will prevent particles coming from the edges to degrade the sample's surface quality during backside polishing. It will also allow optimized visualization of the grinding advancement on one of them. The latter will indeed be observed in cross section view during the physical polishing step with an optical microscope at a $\times 50$ or $\times 100$ magnification. Three sides are therefore polished roughly, using $30 \mu \mathrm{m}$ and 6 $\mu \mathrm{m}$ grade lapping film disks, while the fourth one undergo the same steps, followed by fine polishing steps using $3 \mu \mathrm{m}$ and $1 \mu \mathrm{m}$ grade disks.

The second step features the Si substrate grinding up to a few remaining microns, typically 2 to $5 \mu \mathrm{m}$. This is performed in a semi-automatic fashion by the mechanical polisher. The manual manipulations are here limited to change the grade of the lapping disks when needed and to check for sample alignment from time to time. Most of the substrate is first removed using $30 \mu \mathrm{m}$ disks (up to $\sim 60 \mu \mathrm{m}$ remaining $\mathrm{Si}$ ). The following steps are performed with finer abrasives, with $6 \mu \mathrm{m}(3 \mu \mathrm{m}, 1 \mu \mathrm{m})$ grade disks up to $15 \mu \mathrm{m}(5 \mu \mathrm{m}, 2 \mu \mathrm{m})$ remaining Si. However this is only indicative. The right moment to stop this polishing step is still for the user to decide, knowing that one has to provide the thinnest remaining Si layer in order to reduce chemical etch duration, but that over-polishing might degrade surface quality with appearance of scratches and/or bevel.

After demounting the substrate, the stack is once again cleaned with Acetone and IPA. Then, a metallic Pt layer of typically $\sim 100 \mathrm{~nm}$ thickness is deposited on the 4 sides of the stack as well as on the bottom side of the support substrate. This step is crucial because it avoids side etch of the sample and delamination of the layers of interest during the etch step. To deposit Pt on one side of the stack, we use a dedicated Teflon sample holder allowing the stack to stand still during deposition, and protecting all the sides except the one on which the deposition is performed. The procedure is then repeated for the other sides, and finally $\mathrm{Pt}$ is deposited on the bottom of the support substrate.

The last step of the preparation is the TMAH etch, performed in small volumes and for a short time, to remove the small remaining $\mathrm{Si}$ thickness. If all the previous steps are performed in good conditions, it becomes easy to determine the appropriate moment to stop the etch, since when the silicon is totally removed, the sample colour changes to pink/reddish 
depending on the glue thickness. Even if substantial over-etch was to be performed the expected effects are ought to be small, given the high selectivity over oxide of TMAH etch in our configuration. To furthermore determine the surface quality, one can use an optical microscope to visualize the underneath support substrate patterns by transparency. A typical good surface state is displayed in Fig. 2. The total duration of the whole preparation does not exceed half a day.

\section{RESULTS}

\section{1. SURFACE STATE QUALITY}

One advantage of this preparation method is its high useful surface yield. On Fig. 2 are displayed optical views of a sample before and after TMAH etch step, corresponding respectively to the end of step 2. and the end of step 4. in Fig. 1. On the left image we can observe a light grey surface corresponding to the sample to analyse and a darker patterned region surrounding the former on three sides. The latter corresponds to the surface of the support substrate. Some scratches are visible on the polished sample surface, and the support substrate is not visible by transparency. On the right image, displaying the same sample after TMAH etch, we observe the same structure. The support substrate is now visible through a partially transparent layer corresponding to the layers of interest. On the bottom left corner, some $\mathrm{Si}$ is still remaining from the substrate, and we can still spot some of the scratches present before etching. However, the integrity of the sample seems preserved, i.e. the layers of interest were not delaminated through the etch process. This is attested by the fact that the shape of the thin, transparent film corresponding to the layers of interest matches well with the shape of the light grey region in the left image. Given the extremely small thickness of the remaining layers of interest $(\sim 10 \mathrm{~nm})$, the interference fringes are assumed to be due to a variation of the glue thickness over the sample surface and not to a bevel on the layers of interest themselves. The average throughput of "clean" surface on a $25 \mathrm{~mm}^{2}$ original surface was found to be of $17.5 \mathrm{~mm}^{2}$. This preparation method therefore allows achievement of a $\sim 70 \%$ useful surface by sample, which is largely sufficient for multi-technique analysis.

The other advantage of this method is the high quality of the surface state it can yield. No three dimensional features (such as pyramids issued from TMAH anisotropic etching properties) can be seen on the surface of the layers of interest in Fig. 2, which seems perfectly smooth. This can be further investigated by Scanning Electron Microscope (SEM) and by AFM. As SEM observation confirms the smoothness of the surface, it does not give any additional information on its quality (not shown). AFM mappings give useful information on the topography at small $\left(\sim 1 \mu \mathrm{m}^{2}\right)$ and big scale $\left(\sim 100 \mu \mathrm{m}^{2}\right)$. The acquisition of $10 \times 10 \mu \mathrm{m}$ scans on several samples and at five different spots on the surface yielded an average Root Mean Square (RMS) roughness of $0.228 \mathrm{~nm}$ and a peak to valley maximum distance of $2.383 \mathrm{~nm}$, which is almost as good as an unprepared Si wafer surface. One of these mappings is displayed in Fig. 3. In conclusion, the surface state yielded by our backside preparation method seems to be excellent, with a large percentage of useful, flat and smooth surface. However this would be meaningless if the layers of interest were etched during preparation. The next point we will investigate is thus the amount of remaining etch stop layer after preparation.

\section{2. REMAINING AMOUNT OF ETCH STOP LAYER}


We will first assess the presence of a SiON layer at the surface of the samples. ToFSIMS profiles, such as the one shown in Fig. 4's top-right frame, tell us that the near surface region features a $\mathrm{Si}, \mathrm{N}$ and O-rich region, followed after $\sim 50 \mathrm{~s}$ sputtering by a region dominated by hafnium compounds. This corresponds perfectly to the nominal description of the stack and constitutes therefore a proof of the SiON presence at the surface of the sample. Only ${ }^{30} \mathrm{Si}^{-}$is shown in Fig. 4 because it is the only signal allowing clear separation of the SiON and HfSiON layers. Indeed, in our samples the nominal Si composition of SiON should be $\sim 30$ at $\%$, while that of HfSiON should be $\sim 10$ at $\%$. This provides the two nice intensity plateaus in ${ }^{30} \mathrm{Si}^{-}$intensity profiles as shown in Fig. 4. On the other hand, both oxygen and nitrogen nominal contents do not significantly vary between $\mathrm{SiON}$ and HfSiON in our samples, which does not allow for separation of these layers by monitoring of $\mathrm{SiN}^{-}$or $\mathrm{SiO}_{\mathrm{x}}{ }^{-}$ with ToF-SIMS.

Parallel AR-XPS has also been used to assess the depth repartition of the chemical components in the backside prepared samples. The plot shown in Fig. 5 uses the relative sensitivity to each chemical component with regards to the detection angle, which allows sorting the components as a function of depth. Thanks to the high numbers of angles acquired, the significance of the depth information is improved [13]. In the same way than with ToFSIMS, we observe a phase evolution (due to the vertical stacking of heterogeneous layers) with depth in AR-XPS. There is indeed a strong $\mathrm{Si} / \mathrm{Si}-\mathrm{O}$ signature at the surface of the sample (increasing when using shallower detection angles) and a Hf compound signature deeper in the sample (increasing when using wider angles).

Finally, MEIS spectra (Fig. 6) shows a two-step Si distribution, with a more intense Si signal step starting from the surface of the sample and a less intense signal step at higher depths. This corresponds to successive Si-rich and Si-poor layers, signature of SiON and HfSiON layers respectively.

In order to measure the remaining SiON thickness, we used several calculation methods. With ToF-SIMS, we neglected the near-surface sputter rate variation due to Cs pileup transient and assumed a constant yield in $\mathrm{SiON}$ equal to the bulk $\mathrm{SiO}_{2}$ sputter rate. Then we declared the end of the $\mathrm{SiON}$ layer at ${ }^{30} \mathrm{Si}^{-}$half signal between $\mathrm{SiON}$ and HfSiON region (see Fig. 4). The sputter time necessary to reach this limit is then converted in nanometres using the bulk $\mathrm{SiO}_{2}$ sputter rate obtained with our reference sample. As observed in Fig. 4, there is a slight evolution from sample to sample of the sputter time needed to completely remove the SiON layer. This implies a small variation of the over-etch duration from a sample to another. However, the average thickness of remaining $\mathrm{SiON}$ is therefore found to be of $1.27 \pm 0.25 \mathrm{~nm}$. This is quite close to the nominal SiON thickness of $1.5 \mathrm{~nm}$, notwithstanding the approximation done on constant sputter rate in SiON. This means that even in the worst case of extensive over-etching more than two thirds of the original layer was still left.

MEIS results agree quite well with these conclusions, with an average remaining SiON thickness measured at around $1 \mathrm{~nm}$. These results were obtained by spectra simulation and measurement of Si dose in the surface layer corresponding to SiON. This Si dose was then transformed in layer thickness by assuming a $2200 \mathrm{~kg} \cdot \mathrm{m}^{-3}$ density for SiON (approximated from $\mathrm{SiO}_{2}$ density of ref. [14]). AR-XPS results did not allow straightforward measurement of SiON thickness due to the absence of Si substrate in the backside prepared sample, enhancing the difficulty to setup a proper depth scale.

The backside preparation method we present here is therefore shown to yield excellent surface state without extensive removal of the layers of interest. This allows us to investigate the improvements it brings for analysis of the sample's properties with various characterization techniques. 


\section{3. IMPROVEMENTS IN STACK ANALYSIS WITH VARIOUS TECHNIQUES}

Each technique used in this work presents some limitations, intrinsically related to the physics of the instrument and prohibiting accurate sample characterization. In the particular case of our high-k/metal gate stack, much of the recent research interest has focused on the diffusion of the different elements (in particular lanthanum) throughout the stack during the activation anneal. What matters here is therefore the ability of a technique to resolve the La depth distribution in the most accurate manner possible.

\section{3. 1. ToF-SIMS}

Use of SIMS or ToF-SIMS implies abrasion with a more or less energetic, chemically active or not, primary ion beam. This usually generates two kind of detrimental effects: (i) physical effects, i.e. roughness build-up during analysis or mixing of the near surface layers due to the energetic impact, and (ii) chemical effects, i.e. modification of the chemistry of the near surface layers. The latter can occur because of shallow implantation and adsorption of the primary ion species leading to the creation of a modified surface layer (with active elements such as $\mathrm{O}$ or $\mathrm{Cs}$ ), but also because of the segregation of some elements present in the sample to analyze reacting to the energy deposited on impact (therefore unrelated to the chemical properties of the primary ion species). The latter is well known to happen in samples containing Hf where the latter is expected to be implanted upon impact and to segregate back to the surface, forming a partially modified metallic Hf layer [15]. These modifications of the surface composition and nature during analysis results in dramatic change in both sputter rates and ionization yields, increasing the difficulty to obtain accurate analysis [8, 15]. Fig. 7 shows the profiles obtained by ToF-SIMS on the same high-k/metal gate sample, using frontside and backside analysis.

Both approaches yield similar profiles in the TiN region at least in terms of sputter rate, given the good alignment of the profiles in this region. However there is huge horizontal scale distortion in the right region of the image, corresponding to the HfSiON and beneath layers. This is a direct effect of the Hf segregation during analysis, as discussed above. The same effect is not observed on the backside profile (there is no extensive Hf compound secondary ion tails in the TiN region). This is assumed to be due to the metallic nature of the layer beneath hafnium silicate in the backside approach, avoiding the formation of another (fractional) Hf based metallic layer at the surface of the sample while profiling. Since the extent of the scale distortion is unknown, it would be extremely difficult to perform an accurate scale correction by conventional calculation approaches. Vandervorst et al. [8] have performed some elaborate work on the subject, but they mainly focus on depth scale correction after the end of the hafnium containing layers (in the Si substrate), and not inside or close to those layers. In our case, we want to perform the latter since we want to assess the La distribution in the stack. A very simple depth scale correction has therefore been designed in order to make $\mathrm{HfO}_{2}{ }^{-}$distribution of the frontside profile fit to that of the backside profile. It consists in dividing by 3 the time scale for all points at the right of the vertical line, i.e. all analysis points acquired in the layers beneath the HfSiON layer and in the HfSiON layer itself. As testified by the overlaid backside and corrected frontside profiles in Fig. 8, the Hf compound secondary ion signals fit quite well, assessing the validity of the horizontal scale correction. However, important differences in the depth distributions of the other elements are observed. In particular La distribution in frontside profile is found to be largely erroneous, showing artificial diffusion towards the substrate (grey zone in Fig. 8). This is assumed to be 
due to the second effect of Hf segregation: the modification of sputter/ionization yields for other elements. The latter would be again very difficult to assess without access to reliable depth profile as provided by the backside analysis.

The accuracy of ToF-SIMS analysis on this kind of sample is thus greatly enhanced by using the backside preparation approach proposed in this work, yielding more realist elemental distributions in the stack and allowing quantitative studies to be done (work in progress).

\section{3. 2. MEIS}

MEIS allows matrix effect free analysis, which means the output energy spectra for a particular element will only depend of its depth distribution and its abundance in the stack and not of the surrounding elements. However, the energy of the backscattered ions depends on the masses of the incident ion and the target atom and also on the scattering angle. Thus the mass resolution obviously depends on the energy resolution of the instrumental setup, which in our case is given by $\Delta \mathrm{E} / \mathrm{E} \sim 0.35 \%$. Mass resolution is optimized (1) for the largest scattering angles, (2) by using heavier incident ions $\left(\mathrm{He}^{+}\right.$instead of $\left.\mathrm{H}^{+}\right)$, or (3) by increasing the primary beam energy, but it remains a difficult issue to solve for heavy target elements of similar masses. In the present study, the small mass difference between La and Hf combined to their localisation in the stack makes the spectral separation problematic. In our samples La $(Z=57)$ is placed right on top of $\mathrm{Hf}(Z=72)$. The simulated backscattered spectra displayed in Fig. 9 show that in the frontside analysis configuration the peaks corresponding to La and Hf overlap, which complicates the analysis although geometry was optimized for enhanced peak separation. Since the detector was placed at the largest possible angular position the only solution is then to increase the incident beam energy (see Fig. 9 top left frame), but this would deteriorate energy resolution (and thus depth resolution), which is detrimental to the analysis of such stacks. On the other hand, in the case of the backside approach one can see that the two peaks are clearly separated. This allows diminution of the minimum incident energy needed to resolve both peaks and thus permits a more precise study of the stacks.

Our backside sample preparation would therefore be a crucial advantage for analysis of those samples with MEIS. A complete study of these samples in terms of layer composition and of evolution upon anneal is in progress and will be published soon.

\section{3. 3. AR-XPS}

In the case of XPS or AR-XPS it would be impossible to yield useful information from a frontside analysis of such a stack. The usual depth of information with these techniques is usually of no more than $6 \mathrm{~nm}$ in depth (except if synchrotron sources are used), which prohibits analysis of the layers situated beneath the TiN layer (6.5 nm thick). Removal of the whole TiN layer is not an option, since given the high reactivity of La to oxygen the composition of the stack would change. Deposition of a thinner TiN layer would also modify the stack structure. Indeed the thermal budget seen by the different layers during the Poly-Si deposition and activation annealing would change; and it might also affect the stability of some elements inside the stack. The last option would be to remove only a fraction of the TiN layer by chemical (or plasma) etch. However this would require an extreme control of the etch rate and duration and might deteriorate sample surface quality, making precise analysis of the stack more difficult. Therefore, a promising way to perform stack analysis without modifying the sample structure or composition seems to be the backside approach developed in this work. 


\section{CONCLUSION}

In this work we present an original, two step backside preparation method for samples featuring ultra-thin (nanometer thick) etch stop layer. The method was shown to be simple, enabling routine (although not fully automatic) workflow. It was shown to provide high useful surface ratio with smooth and flat surface for analysis, with little deterioration of etch stop layer.

We applied this method to ultra-thin high-k/metal gate stacks for sub-32 nm node devices and analyzed samples with ToF-SIMS, MEIS and parallel AR-XPS. Important improvements in accuracy of sample analysis were shown to be brought by the backside preparation we developed, compared to standard frontside analysis. 


\section{REFERENCES}

1. F. Laugier, J.M. Hartmann, H. Moriceau et al., Appl. Surf. Sci. 231-232, 668 (2004)

2. C. Hongo, M. Takenaka, Y. Kamimuta et al., Appl. Surf. Sci. 231-232, 594 (2004)

3. C. Hongo, M. Tomita and M. Takenaka, Appl. Surf. Sci. 231-232, 673 (2004)

4. J. Sameshima, R. Maeda, K. Yamada et al., Appl. Surf. Sci. 231-232, 614 (2004)

5. J. Bennett, M. Quevedo-Lopez and S. Satyanarayana, Appl. Surf. Sci. 252, 7167 (2006)

6. T. Hantschel, C. Demeulemeester, A. Suderie et al., in Electron Crystallography for Materials Research and Quantitative Characterization of Nanostructured Materials, edited by P. Moeck, et al. (Materials Research Society, Warrendale, 2009), pp. 185

7. T. Conard, W. Vandervorst, H. De Witte et al., Appl. Surf. Sci. 231-232, 581 (2004)

8. W. Vandervorst, J. Bennett, C. Huyghebaert et al., Appl. Surf. Sci. 231-232, 569 (2004)

9. M.J.P. Hopstaken, J.C. Cabral, D. Pfeiffer et al., AIP Conf. Proc. 1173, 94 (2009)

10. O. Tabata, R. Asahi, H. Funabashi et al., Sensors and Actuators A: Physical. 34, 51 (1992)

11. N. Baboux, J.C. Dupuy, G. Prudon et al., J. Cryst. Growth. 245, 1 (2002)

12. J.F. Ziegler, J.P. Biersack and U. Littmark, The Stopping and Range of Ions in Solids. (Pergamon Press New York, 1985)

13. B. Pelissier, A. Beaurain, J.P. Barnes et al., Microelectron. Eng. 85, 1882 (2008)

14. K.E. Petersen, Electron Devices, IEEE Transactions on. 25, 1241 (1978)

15. W. Nieveen, B.W. Schueler, G. Goodman et al., Appl. Surf. Sci. 231-232, 556 (2004) 


\section{FIGURE CAPTIONS}

Fig. 1. Schematic representation of the different steps in the backside preparation process. 1 . Sample is fixated on a support substrate of approximately same size, and the four sides of the stack are polished. 2. Si substrate is grinded with diamond lapping film disks of varying grade up to 2 to $5 \mu \mathrm{m}$ remaining Si. 3. Pt protective layers are deposited on the four sides and on the bottom of the support substrate. 4. Remaining $\mathrm{Si}$ is etched in TMAH.

Fig. 2. Optical image of a sample before (left) and after (right) chemical etch step, snatched from several $\times 2.5$ magnification snapshots. Patterns are clearly visible by transparency, except on the bottom left corner where $\mathrm{Si}$ is still remaining. Interference fringes are assumed to be related to inhomogeneity of the glue thickness (given that the layer of interest thickness is less than $15 \mathrm{~nm}$ ).

Fig. 3. Example of a $10 \times 10 \mu \mathrm{m}$ AFM scan of a sample surface after TMAH etch. The given RMS and Zrange (Peak to valley maximum distance) values are averaged over five acquisitions on the same sample at different locations.

Fig. 4. ${ }^{30} \mathrm{Si}^{-}$distribution in the ToF-SIMS backside approach profiles of two similar high$\mathrm{k} / \mathrm{metal}$ gate samples. The top right frame shows the succession of the layers in the profile of one of the samples through ${ }^{30} \mathrm{Si}^{-}, \mathrm{HfO}_{2}{ }^{-}$ant $\mathrm{TiN}^{-}$secondary ions.

Fig. 5. Relative depth plot of chemical components for a backside prepared sample obtained by AR-XPS.

Fig. 6. MEIS energy spectrum recorded in the Si region with $\mathrm{He}^{+} 100 \mathrm{keV}$ incident at $40^{\circ}$ and using a $105^{\circ}$ detection angle. The highest measured energy position (black arrow) corresponds to ions scattered from surface atoms while the vertical line indicates the limit between SiON and HfSiON contributions.

Fig. 7. ToF-SIMS profiles of the same high-k/metal gate sample, with both frontside and backside approaches. The $\mathrm{x}$ scale of the backside profile has been inverted for comparison purpose. A vertical dotted line indicates the virtual limit between the titanium nitride region (left) and the lanthanum oxide/hafnium silicate/silicon oxynitride region (right). A corrected frontside profile is displayed in the bottom frame.

Fig. 8. Overlaid backside and corrected frontside ToF-SIMS profiles of the same high-k/metal gate sample, focused on the high-k layer.

Fig. 9. Frontside and backside simulated MEIS spectra in the Hf / La region, with a $200 \mathrm{keV}$ $\mathrm{He}^{+}$primary beam incident at $21^{\circ}$, and a $131^{\circ}$ detection angle. The structure of the simulated stack is: $\mathrm{TiN}(6.5 \mathrm{~nm}) / \mathrm{La}_{2} \mathrm{O}_{3}(0.4 \mathrm{~nm}) / \mathrm{HfSiON}(1.7 \mathrm{~nm}) / \mathrm{SiON}(1.5 \mathrm{~nm}) / \mathrm{Si}$ (substrate) in the case of the frontside approach, and: $\mathrm{SiON}(1.5 \mathrm{~nm}) / \mathrm{HfSiON}(1.7 \mathrm{~nm}) / \mathrm{La}_{2} \mathrm{O}_{3}(0.4 \mathrm{~nm}) / \mathrm{TiN}(6.5 \mathrm{~nm})$ in the case of the backside approach. Simulation was done with a home-made software, taking into account straggling and neutralization effects. Hf peak intensities were aligned in energy to allow better visualization of the differences between the two approaches in terms of peak separation power. Insert shows the simulated spectra with a $400 \mathrm{keV} \mathrm{He}^{+}$primary beam incident at $21^{\circ}$, and a $131^{\circ}$ detection angle. 


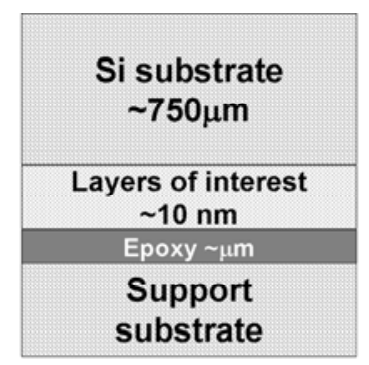

1. Bounding to support substrate



2. Grinding on aumatic polisher

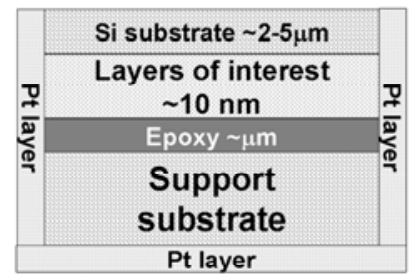

3. Deposition of $\mathrm{Pt}$ protective layer

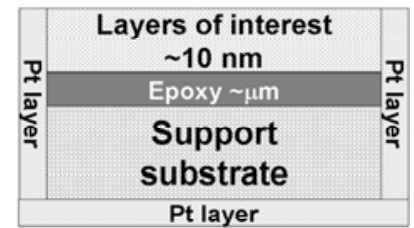

4. TMAH wet etching removal of remaining $\mathrm{Si}$

Figure 1

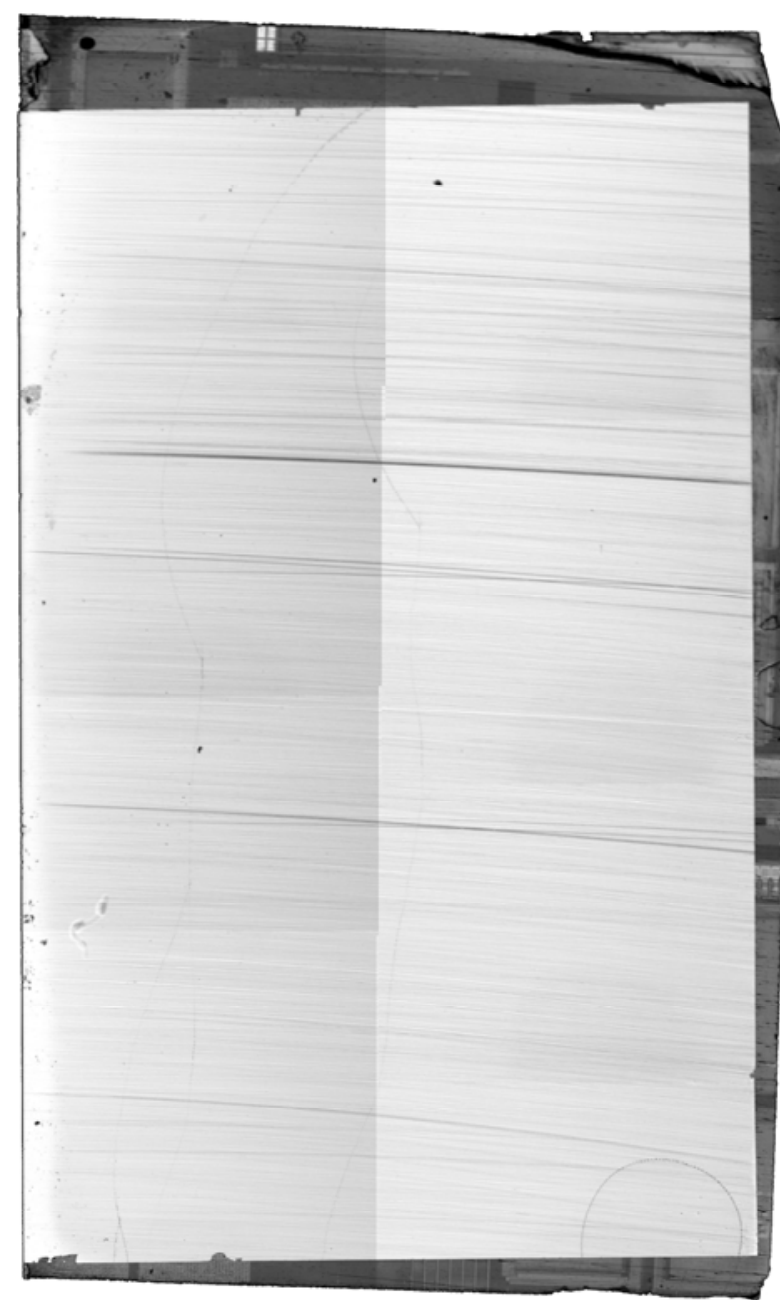

a) Before TMAH etch

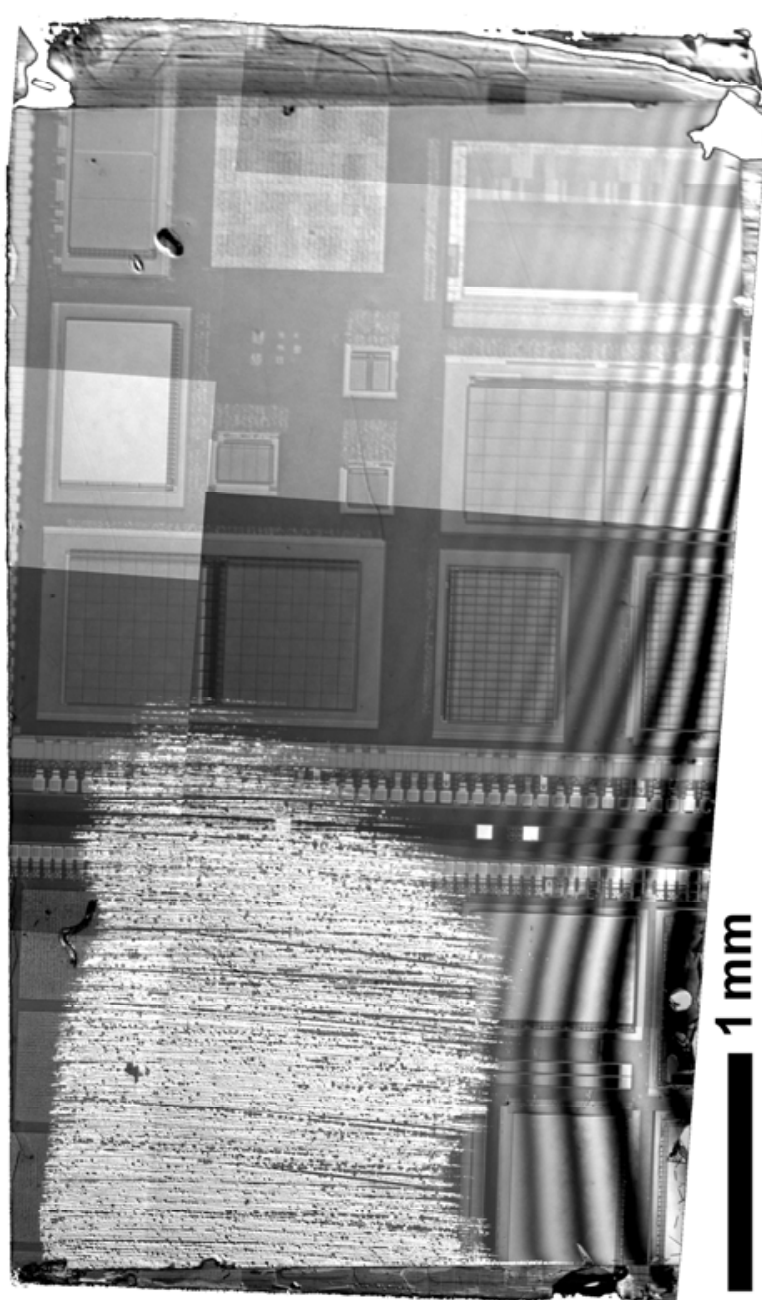

b) After TMAH etch

Figure 2 


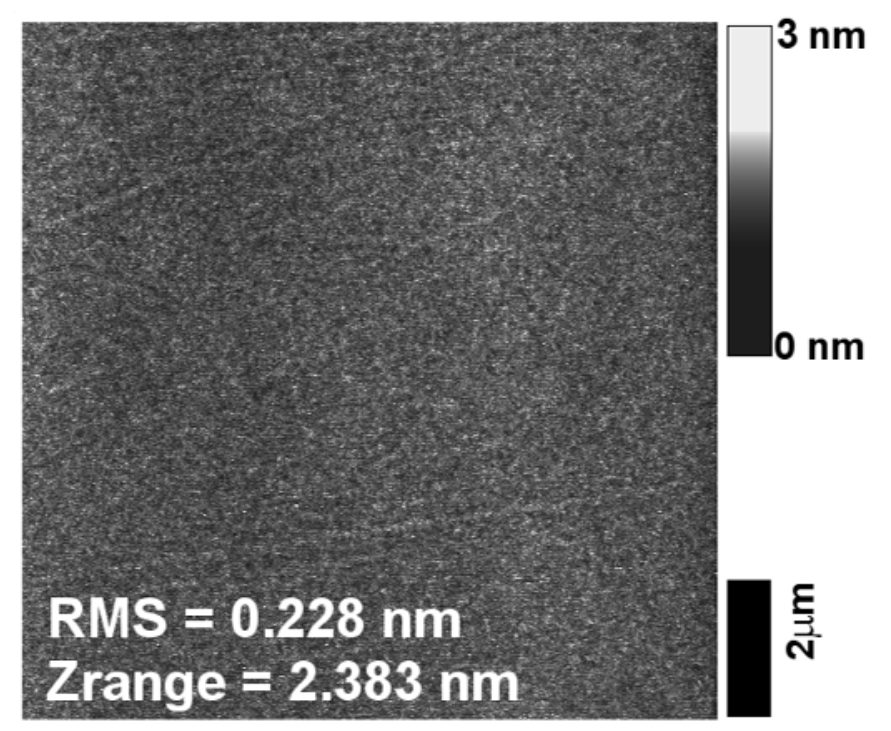

Figure 3

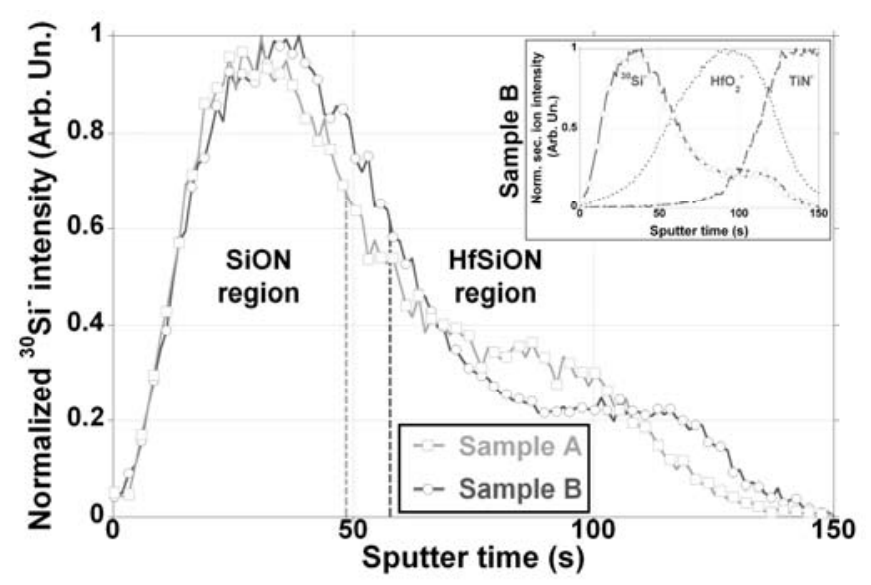

Figure 4



Figure 5 


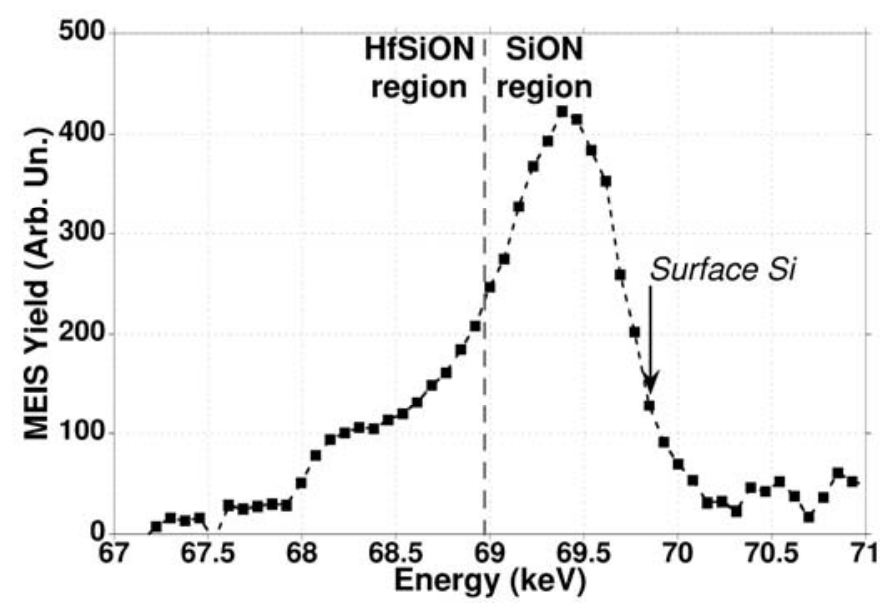

Figure 6

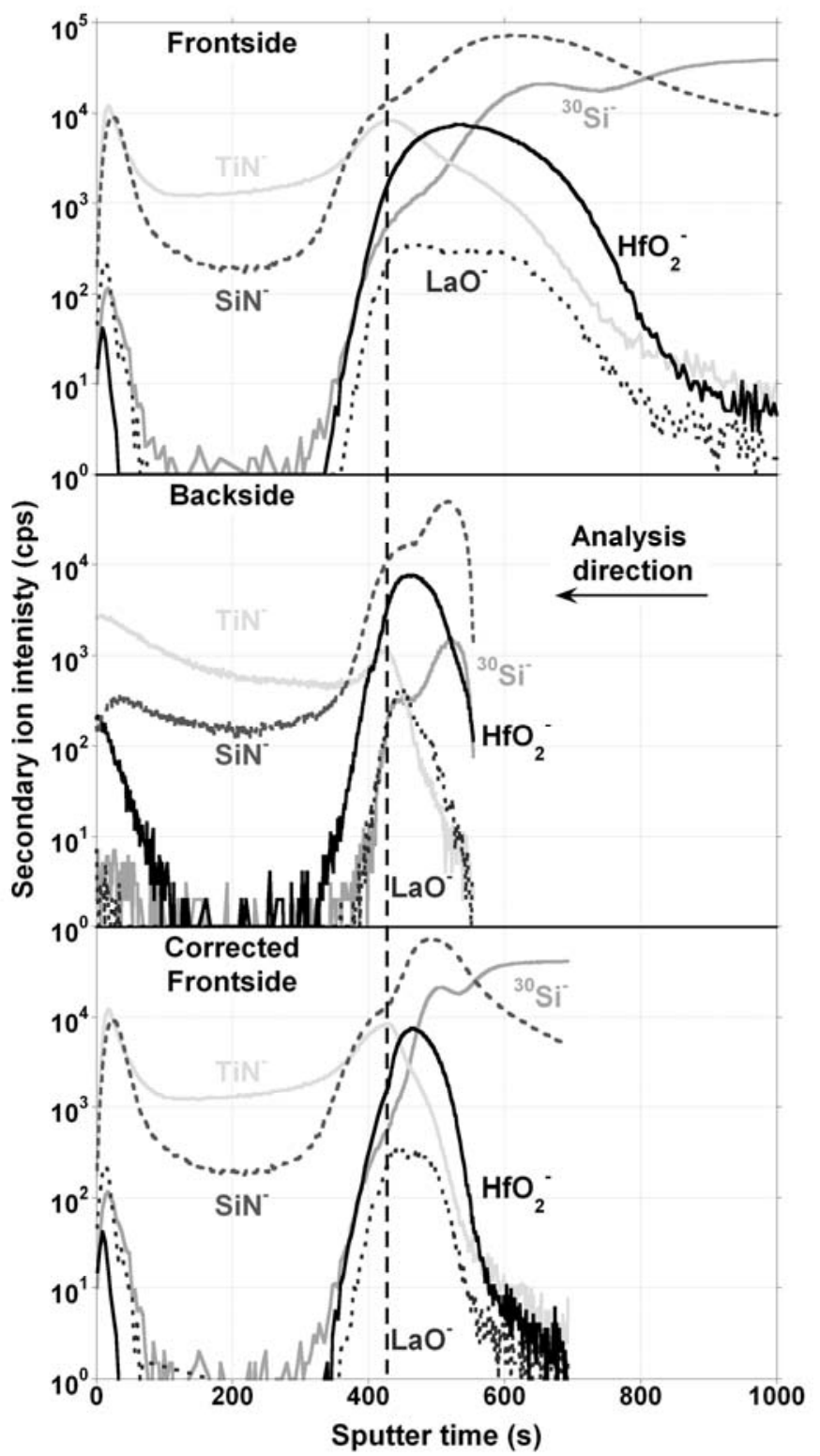

Figure 7 


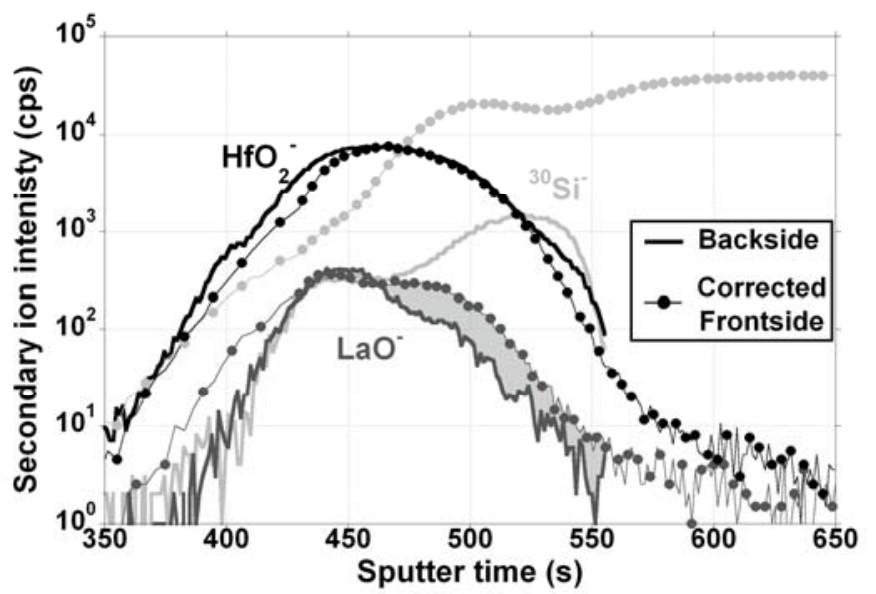

Figure 8

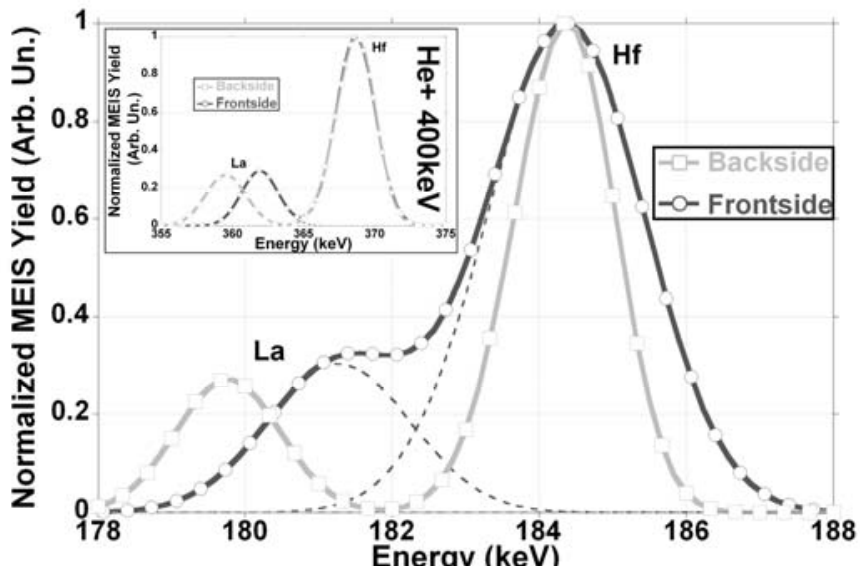

Figure 9 